\title{
A Methodology for Characterizing Fault Tolerant Switched Reluctance Motors Using Neurogenetically Derived Models
}

\author{
Lee A. Belfore, II, Senior Member, IEEE, and Abdul-Rahman A. Arkadan, Fellow, IEEE
}

\begin{abstract}
This paper examines the feasibility of using artificial neural networks (ANNs) and genetic algorithms (GAs) to develop discrete time dynamic models for fault free and faulted switched-reluctance-motor (SRM) drive systems. The results of using the ANN-GA-based (neurogenetic) model to predict the performance characteristics of prototype SRM drive motor under normal and abnormal operating conditions are presented and verified by comparison to test data.
\end{abstract}

Index Terms-Fault tolerance, finite-element methods, genetic algorithms, neural networks, synchronous motors.

\section{INTRODUCTION}

$\mathbf{T}$ HE WORK presented here examines the feasibility of employing artificial-neural-network (ANN) models that are constructed by using genetic algorithms (GAs) to model the performance characteristics of switched-reluctance-motor (SRM) drive systems during normal and abnormal operating conditions. The modeling of these conditions requires the use of coupled magnetic and state space/lumped parameter circuit models of the machine and the associated converter [1]. However, a drawback with this approach is that one needs to fully repeat the analysis for any changes in the system topology, loading, or fault conditions to characterize the motor drive system. On the other hand, ANN-built models, due to their interpolation property can avail that.

Feedforward backpropagation ANNs have shown promise in modeling many electromechanical applications (see, for example, [2]-[4]) The work of reference [2] investigated the use of ANNs to detect faults in induction motors. ANN inputs included the motor speed, current, and the three quadratic functions of the speed and current. The models developed in [3], [4] present a feedforward ANN to control position and speed in dc motors. These models have several features including the use of ANN-projected outputs being delayed and fed back to the ANN inputs.

The purpose of this paper is to present a methodology that can be used to construct an ANN capable of modeling the dynamics of SRM drive systems, or any dynamical system. Using ad-hoc techniques to find good ANN architectures consume sig-

Manuscript received March 20, 2000; revised January 18, 2002.

L. A. Belfore is with the Department of Electrical and Computer Engineering, Old Dominion University, Norfolk, VA 23529 USA (e-mail: lbelfore @ odu.edu)

A.-R. A. Arkadan is with the Department of Electrical and Computer Engineering, Marquette University, Milwaukee, WI 53201 USA (e-mail: A.A.Arkadan@marquette.edu).

Publisher Item Identifier S 0885-8969(02)X12866-0. nificant engineering effort and were unsuccessful for solving the problem described in this paper. As a result, GAs were employed to search for a suitable ANN.

This paper is organized into six sections, including an introduction, a presentation of the indirectly coupled finite-elements/state-space (FE/SS) modeling environment, the modeling approach, the neurogenetic (GA based ANN model) used in this work, a presentation of results and conclusions.

\section{INTEGRATED FINITE- ELEMENTS/STATE-SPACE MODELING ENVIRONMENT}

In this section, the details of generating training data sets, for the SRM drive system and ANN networks discussed in this paper, are presented. Most modern electromagnetic (EM) systems involve nonlinear external circuit modules that include solid-state switching devices in addition to the EM device that comprises nonlinear magnetic materials. The magnetic and electrical circuits are interacting during normal operating conditions. This interaction (coupling) becomes more evident during abnormal (fault) conditions. Accordingly, the coupling between the nonlinear magnetic and electric circuits should be accounted for in the analysis when predicting the performance characteristics of a system, especially when the system is under fault conditions.

As can be seen in the recent literature, the coupling effects can be incorporated in the analysis directly or indirectly. In the direct approach, coupling is accounted for by adding the external circuit currents as unknowns directly into the finite-element matrix equation [5], or through the modeling of the circuit elements with zero-dimensional finite elements [6]. The direct approach requires extensive computational time and memory storage space to account for motion and magnetic material nonlinearities.

In the indirect approach, a series of nonlinear field solutions is used, assuming a known current excitation, to determine the magnetic device lumped parameters. Next, these parameters are used with the external circuit electrical equations to predict the performance characteristics of the system [7]. However, when studying faults, the currents exciting the nonlinear magnetic device are unknown as in the case of an SRM drive system under internal or external fault conditions. In these cases, the instantaneous values of the machine lumped parameters, which are the windings self and mutual inductances, continuously vary due to saturation caused by the fault currents, the change in the rotor speed and the change in the topology of the external circuits. 
As stated earlier, a modeling environment based on using ANNs and GAs to predict the performance characteristics of faulted SRM drive systems is presented. As detailed below, the ANNs are applied for their well-known interpolation capabilities for highly nonlinear systems and the AUTHOR, PLEASE DEFINE THIS ACRONYM EAs are employed for their ability to search a complex structural and parametric space as necessary to find good ANN solutions. Furthermore, an integrated FE/SS modeling approach is used to generate training data sets for the ANN model of an SRM drive system. The FE/SS approach presented is indirect and is based on the use of nonlinear magnetic field solutions and state-space models, including the speed equation.

The two-dimensional equation governing the field in the magnetic device after neglecting the displacement and induced current terms can be expressed as

$$
\frac{\partial}{\partial x}\left(\nu \frac{\partial A}{\partial x}\right)+\frac{\partial}{\partial y}\left(\nu \frac{\partial A}{\partial y}\right)=-J_{e}
$$

where

A z-component of the magnetic vector potential (mvp);

$\nu \quad$ magnetic reluctivity;

$J_{e} \quad \mathrm{z}$-component of the excitation current density vector; Meanwhile, the state space model describing the motor-drive system is as follows:

$$
\underline{V}=\underline{R I}+\underline{L} \frac{d \underline{I}}{d t}+\omega_{m} \frac{d \underline{L}}{d \theta} \underline{I}
$$

where

I state variables of the system that are the machine phase currents $i_{a}, i_{b}$, and $i_{c}$;

$\underline{L} \quad$ SRM self and mutual inductances;

$\underline{V}$ machine terminal voltages to the external circuits (Fig. $1 b)$;

$\underline{R}$ diagonal matrix representing the phase resistances;

$\theta$ rotor position;

$\omega_{m}$ rotor speed.

In addition, the equation used to solve for the rotor speed $\omega_{m}$ is given as

$$
\frac{d \omega_{m}}{d t}=\frac{1}{J}\left(T_{e m}-B \omega_{m}-T_{L}\right)
$$

where

$J \quad$ inertia of the rotor;

$T_{e m}$ developed electromechanical torque;

$B$ coefficient of viscous friction;

$T_{L} \quad$ load torque.

Based on this approach, the unknown values of the excitation currents, machine inductances, and rotor speed are continuously updated during a sustained fault condition using an iterative technique.

\section{MODELING APPROACH}

To study the coupled problem in an SRM drive system during sustained fault conditions, an iterative approach is used. This approach is based on the use of 2D FE analysis, (1), in conjunction with the energy perturbation method for inductance calculation and results from SS models, (2) and (3). A flowchart outlining

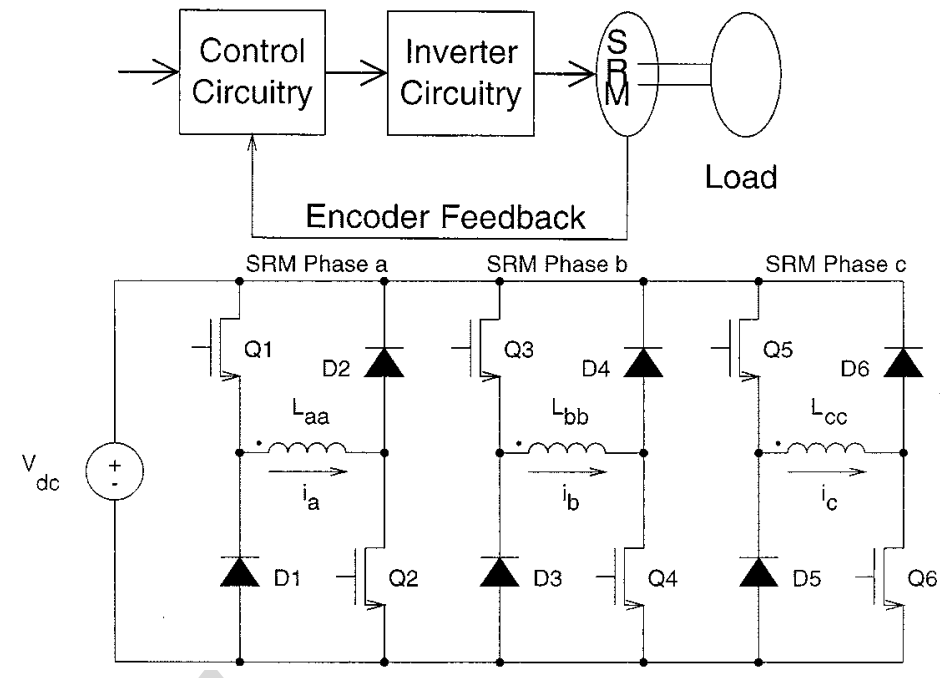

Fig. 1. SRM drive system.

this approach is shown in Fig. 2. The step-by-step procedure is outlined as follows [2]:

Step 1) Perform a series of FE field solutions corresponding to a set of rotor positions covering a period of $\left[0^{\circ}\right.$ to $180^{\circ}$ ] using intervals of $2^{\circ}$ mechanical each to determine the inductances for a no fault load condition.

Step 2) Using the computed inductances in the SS model, a fault condition is simulated. The SS model equations are integrated until the fault reaches steady state.

Step 3) The currents from this analysis are then used in the FE model to predict a new set of updated inductances.

Step 4) These updated inductances are next used in the SS model to predict a new set of fault currents.

Step 5) Repeat Steps 2 through 4 until a convergence criterion is satisfied.

The normalized rotor speed computed from the SS model is used as the criterion for convergence during the fault condition. The normalized speed change from one iteration to the next is as follows:

$$
\frac{\omega_{i}-\omega_{i+1}}{\omega_{s s}} \leq \epsilon
$$

where

$\omega_{i} \quad$ speed at iteration $i$

$\omega_{i+1}$ speed at iteration $i+1$;

$\omega_{s s}$ steady-state speed prior to the onset of the fault.

If the normalized speed change is less than a convergence tolerance $\epsilon$, the resulting fault condition is considered to have converged.

The implementation of Step 1 of this iterative approach required the use of FE analysis. Furthermore, the implementation of Steps 1 and 3 required the calculation of the inductance values. This was performed by using an energy and current perturbation approach applied to numerical magnetic field solutions. This approach was developed and experimentally verified in earlier works [8], [9]. Also, the implementation of Step 3 required the use of the calculated inductances in the state model of (2). This was accomplished by representing the machine self 
and mutual inductances by Fourier series-type expressions of the form

$$
L_{j k}=c_{o}+\sum_{n=1}^{N H}\left[c_{n} \cos (n \theta-\phi)\right]
$$

where

$$
\begin{array}{ll}
c_{O} & \text { dc value of the inductance; } \\
c_{n} & \text { magnitude of the } n \text {th harmonic; } \\
\phi & \text { phase shift; } \\
N H & \text { limit of the harmonic order. }
\end{array}
$$

\section{Neurogenetic Model}

In this work, a discrete time model for an SRM drive system is constructed using ANNs. ANN's are employed for their well known interpolation capabilities. A properly designed and trained ANN is capable of interpolating for cases in which it was not trained. This is in contrast to the conventional approach of [1] where the FE and SS analysis must be performed for each new operating condition. Furthermore, the ANN can be trained directly from actual SRM measured data or from simulation data. Relevant ANN model design considerations are the selection of inputs, the number of hidden layers, the number of neurons in each hidden layer and training parameters. For temporal modeling, the number of time delayed samples for inputs must be selected as well. The ANN model used in this work is illustrated in Fig. 3. The model assumes one ANN, N1, models the dynamics of the SRM while the second ANN, N2, maps the state variables and inputs to the predicted torque. N1 is trained as a feedforward ANN that computes state-variable updates for current $(\underline{I})$, position $\left(q_{m}\right)$ and speed $\left(\omega_{m}\right)$. The position is represented by two parameters that are the sin and cosine of the mechanical phase (or rotor position) rather than $\theta$. Decoupling the rotor position in this fashion provides two benefits. First, the model training is not confounded by discontinuities in the mechanical phase because simulation models report $\theta \in[0,2 \pi)]$. Second, if the position were the continuous linear phase since the beginning of the simulation, training may be overly biased to synchronize the ANN outputs with $\theta$, rather being less capable of modeling true dynamical behaviors. Ad-hoc techniques were employed, without success, to find an acceptable ANN model.

GAs are algorithms based on an analogy with evolution observed in nature. GAs are optimization techniques that perform a directed, randomized search of the problem space to find optimal and nearly optimal solutions [10], [11]. Due to the randomized nature of the search, GAs can search for solutions without making assumptions about the structure of the best solution. Furthermore, GAs are relatively immune to becoming trapped in local minima, because of the randomized search process. The genetic algorithm uses an averaging operator to combine parent organizms. This operator was selected because organism genomes specify coarser structural aspects of the organism. This operator was selected because of the parametric nature of the ANN structure. Furthermore, the probability of a mutation in the child is $5 \%$. The mutation operator consists of Gaussian additive noise for the number of layers in the

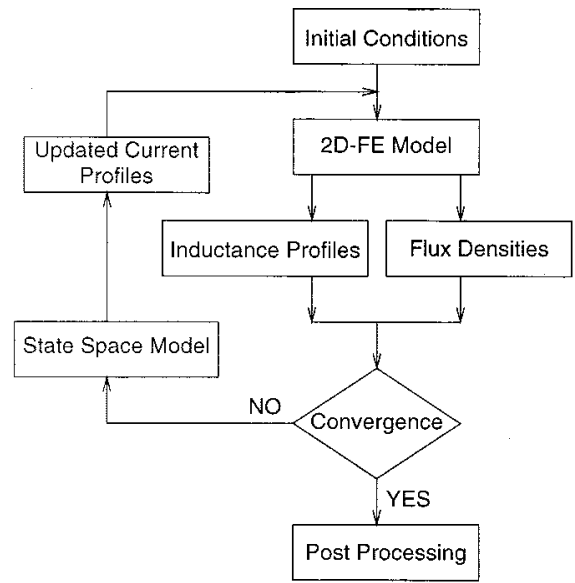

Fig. 2. Flowchart of iterative finite element approach.

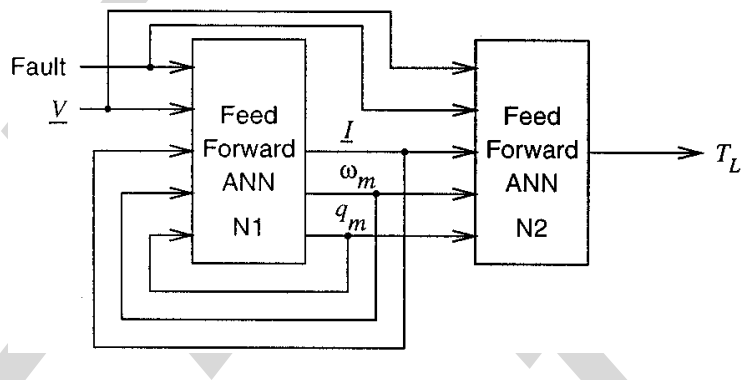

Fig. 3. ANN architecture for modeling SRM.
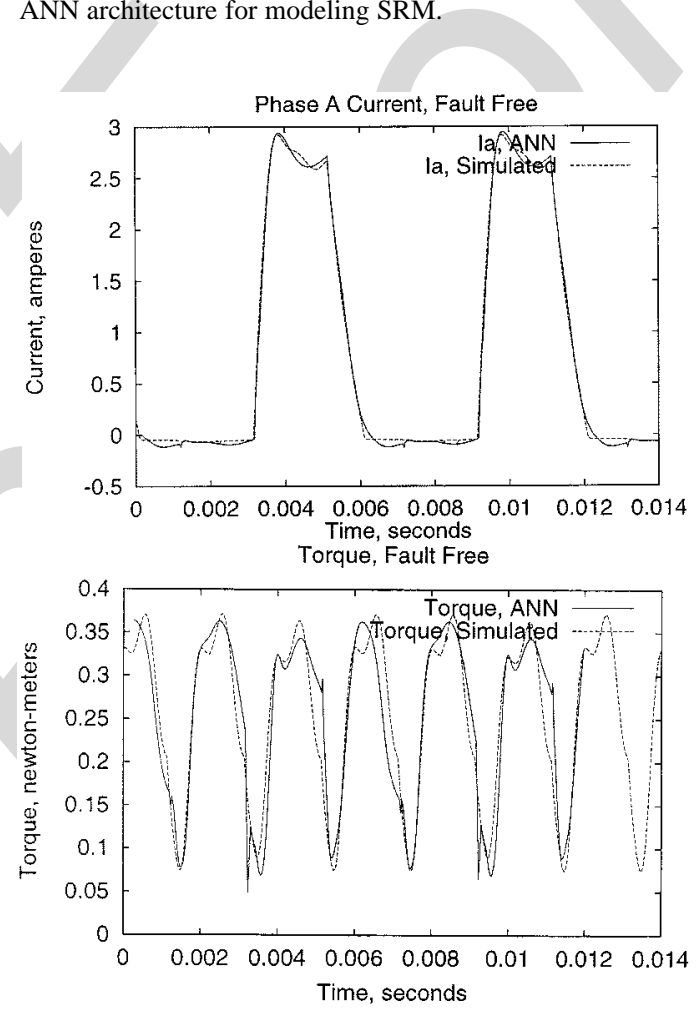

Fig. 4. Normal (no fault) condition, phase a current.

network, the number of neurons in each layer, the number of delayed samples for each input, and the training time. For the learning rate, the mutation was constructed so that the range of the mutated values remained in the range of $[0,1]$. The 
following equation describes the mutation for the learning rate and momentum:

$$
v_{\text {new }}= \begin{cases}\left(v_{\text {old }}\right)^{N(1,0.1),} & v_{\text {old }} \leq 0.5 \\ 1-\left(1-v_{\text {old }}\right)^{N(1,0.1),} & v_{\text {old }}>0.5\end{cases}
$$

where $N(1,0.1)$ is a normal random number with a mean of one and a standard deviation of 0.1 . This relation ensures that a value in the range $[0,1]$ remains in that range and that the affect on the variable is symmetric with respect to the midpoint of the range of values. The GA is used to determine the structure of the $\mathrm{ANN}$ and the ANN is trained using backpropagation to perform a local search.

In order to determine how well prospective ANN's model the SRM, a fitness function is defined. The fitness function is designed to have two fitness tiers. The first tier selects ANNs that have low open-loop error. The second tier selects ANNs with low closed-loop error. The two tier strategy works under the premise that a network having a low closed-loop error will also have a low open-loop error. The fitness is computed according the following formula:

$$
\begin{aligned}
F= & -e^{-0.24(G-1)} \\
& \cdot\left(\log (T)+0.5 \log \left(N_{W}\right)+\log \left(N_{N}\right)+2 \log \left(N_{L}\right)\right) \\
& -4 \log \left(E_{O}\right)-10 \log \left(E_{C}\right)
\end{aligned}
$$

where

$F \quad$ fitness;

$G \quad$ current generation;

$T$ number of training epochs;

$N_{W}$ total number of weights in the network;

$N_{N}$ total number of neurons in the network;

$N_{L} \quad$ number of layers in the network;

$E_{O} \quad$ open-loop error;

$E_{C} \quad$ closed-loop error.

The decay as a function of generation for training and structural resources is set so that after ten generations, these contributions are one tenth the initial contribution. For each generation, the population is simulated and the fitness is determined for each individual using (7) followed by a ranking based on fitness. The subsequent generation is determined from the present generation using a process that favors better solutions, yet enables variations in prospective solutions to search the space.

\section{Results of a CASE Study}

The neurogenetic models described earlier were used to model the SRM drive system of Fig. 1(a) under normal and fault conditions. Using the GA described before, the fittest ANN was found in the 71st generation. The average open-loop mean-square error for this network is 0.00137 , an average closed-loop mean-square error is 0.00720 and a resulting fitness of 75.72. This ANN has two hidden layers of 13 and 18 neurons, respectively. For each input, the number of time-delayed samples for each input averaged approximately 4 samples per input. The ANN was trained with a learning rate $\alpha=0.11$, a learning momentum $\beta=0.16$ for 14 epochs.

The neurogenetic model was used to predict the performance characteristics of the SRM drive system of Fig. 1(a) under the following:

Case 1) Normal (No Fault) Condition in Fig. 4;

Case 2) Transistor Fault Condition in Fig. 5;

Case 3) Partial Phase Fault condition in Fig. 6

It should be noted here that Case \#2 corresponds to the condition when transistor Q2 fails and results in the loss of phase (b) of the SRM. Meanwhile, Case \#3 corresponds to a partial failure of phase (b) resulting from a short in the winding of phase (b). Sample results for current waveforms and torque profiles are given in Figs. 4-6, for these cases, respectively. In these figures, the ANN results are shown and compared to FE simulation results obtained from the conventional models described in [1]. Also, a comparison of RMS measures from the ANN, the FE, and test data is given in Table I. An inspection of the waveforms in Figs. 4-6 as well as the results in Table I reveal good agreement between the three sets of data. Accordingly, it is demonstrated that the GA-based ANN (neurogenetic) models can be used for the characterization of motor drive systems under normal and fault conditions.

While the agreement and general characterization are good, errors in the mappings are of concern. Since the ANN mapping is trained using backpropagation, after training, small mapping errors are present. An additional complication is that the ANN represents the SS mapping relation, where errors in the state can perturb subsequent state updates. Indeed, the torque shows some sensitivity at specific rotor positions. In particular, the model in this case study is sensitive to the current switching on and off in phase a. The sensitivity is likely a result of the decoupling of the torque prediction from the rest of the mappings. The currents used in training the torque are not the currents predicted by the ANN mapping, but rather are the currents taken from the original FE/SS simulation. Such excursions are seen in the torque curves in Figs. 4(b) and 5(b)

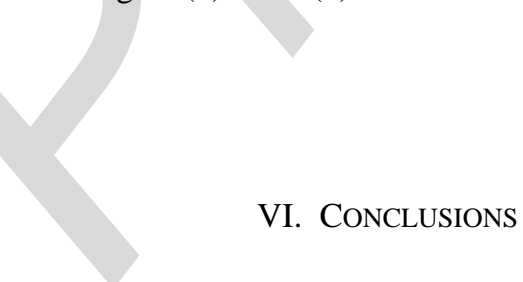

This work showed the feasibility in constructing a multilayered feedforward ANN using GA methodologies to model the discrete time dynamics of faulted and fault-free SRM drive systems. The GA search is run for 20 generations and included a search of the number of time-delayed samples for each input, the number of layers, the number of neurons in each layer, the training duration and training rates. A local search, in the form of the backpropagation learning algorithm, is performed on the organizm defined by these quantities. The performance of fittest ANN model was excellent, in comparison to the simulated and test results. 

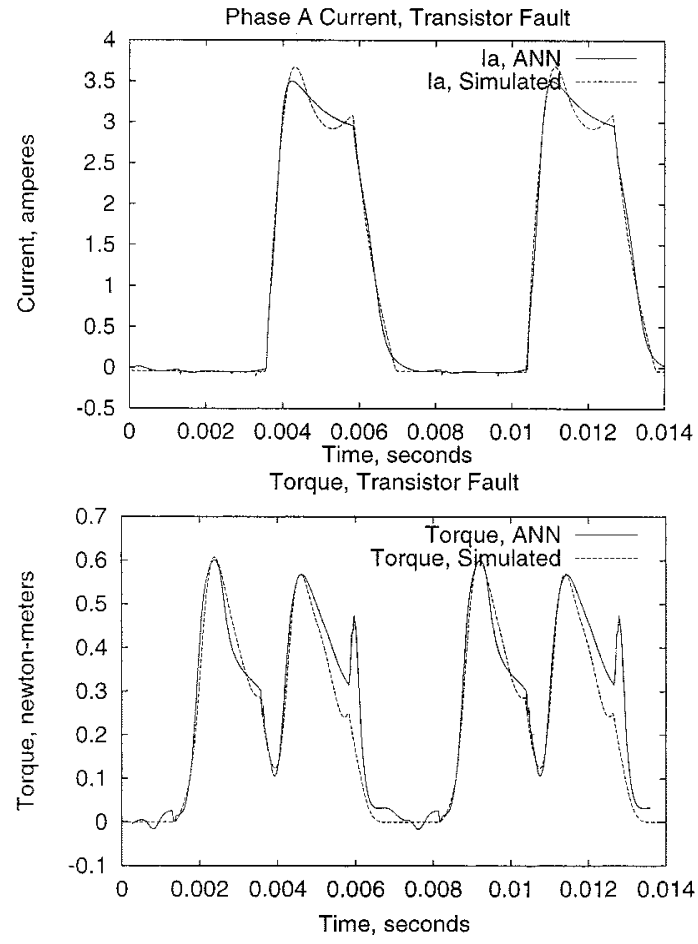

Fig. 5. Transistor fault condition, phase a current.
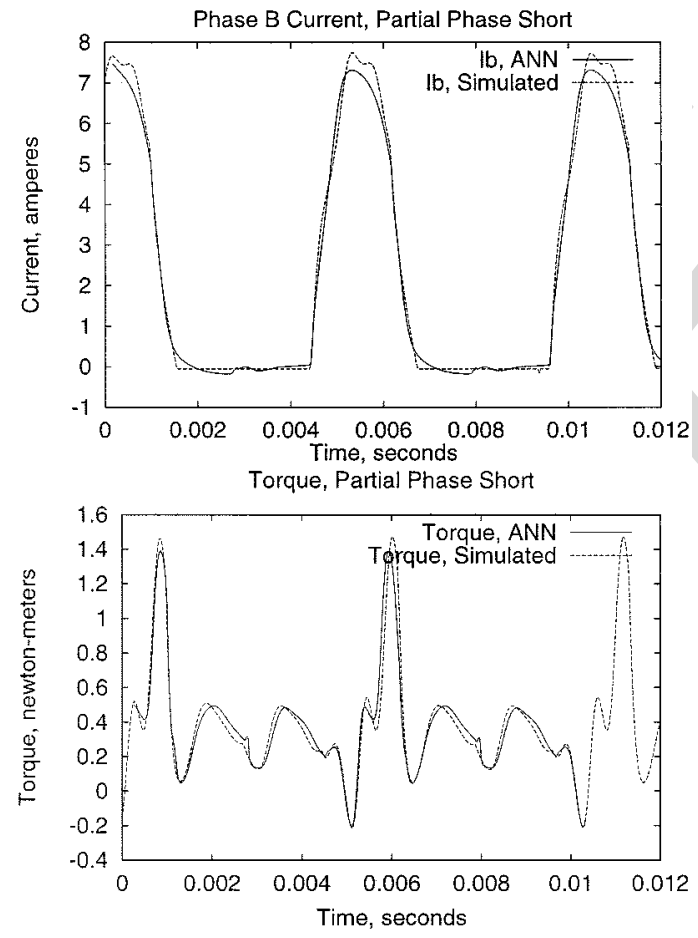

Fig. 6. Partial phase short condition, phase b current.

\section{REFERENCES}

[1] A. A. Arkadan and B. W. Kielgas, "The coupled problem in switched reluctance motor drive systems during fault conditions," IEEE Trans. Magn., vol. 30, pp. 3256-3259, 1994.

\section{Need month for REF. 1}

[2] M.-Y. Chow and S. O. Yee, "Using neural networks to detect incipient faults in induction motors," J. Neural Network Comput., pp. 26-32, Winter 1991.
TABLE I

COMPARISON OF ANN AND MEASURED RMS VALUES

\begin{tabular}{c|c||c|c|c}
\hline \multicolumn{2}{c||}{} & \multicolumn{3}{c}{ Motor status } \\
\cline { 3 - 5 } \multicolumn{2}{c|}{ Model } & $\begin{array}{c}\text { Fault } \\
\text { Free }\end{array}$ & $\begin{array}{c}\text { Transistor } \\
\text { Fault }\end{array}$ & $\begin{array}{c}\text { Phase } \\
\text { Short }\end{array}$ \\
\hline \hline \multirow{2}{*}{$\begin{array}{c}I_{a} \\
(\text { A,RMS })\end{array}$} & Measured & 1.080 & 1.280 & N/A \\
\cline { 2 - 5 } & Simulated & 0.968 & 1.147 & 1.077 \\
\cline { 2 - 5 } & ANN & 0.963 & 1.149 & 1.068 \\
\hline \hline \multirow{3}{*}{$\begin{array}{c}I_{b} \\
(\text { A,RMS })\end{array}$} & Measured & 1.080 & 0.0 & N/A \\
\cline { 2 - 5 } & Simulated & 0.989 & -0.021 & 2.165 \\
\cline { 2 - 5 } & ANN & 1.025 & 0.006 & 2.109 \\
\hline \hline \multirow{2}{*}{$\begin{array}{c}\text { Torque } \\
(\mathrm{Nm}, \mathrm{RMS})\end{array}$} & Measured & 0.217 & 0.217 & N/A \\
\cline { 2 - 5 } & Simulated & 0.253 & 0.236 & 0.364 \\
\cline { 2 - 5 } & ANN & 0.253 & 0.236 & 0.364 \\
\hline \hline \multirow{3}{*}{$\begin{array}{c}\omega m \\
(\mathrm{rad} / \mathrm{s})\end{array}$} & Measured & 261.8 & 212.3 & N/A \\
\cline { 2 - 5 } & Simulated & 261.8 & 230.1 & 306.2 \\
\cline { 2 - 5 } & ANN & 261.9 & 230.1 & 306.0 \\
\hline
\end{tabular}

[3] M. A. El-Sharkawi, A. A. El-Samahy, and M. L. El-Sayed, "High performance drive of dc brushless motors using neural network," IEEE Trans. Energy Conversion, vol. 9, pp. 317-322, June 1994.

[4] S. Weerasooriya and M. A. El-Sharkawi, "Identification and control of a dc motor using back-propagation neural networks," IEEE Trans. Energy Conversion, vol. 6, pp. 663-669, Dec. 1991.

[5] F. Piriou and A. Razek, "Coupling of saturated electromagnetic systems to nonlinear power electronic devices," IEEE Trans. Magn., vol. 24, pp. 274-277, Jan. 1988.

[6] A. A. Arkadan and R. V. VanderHeiden, "Three dimensional nonlinear finite element modeling of a voltage source excited transformer feeding a rectifier load," IEEE Trans. Magn., vol. 28, pp. 2265-2267, Sept. 1992.

[7] A. A. Arkadan, T. M. Hijazi, and N. A. Demerdash, "Computer-aided modeling of a rectified dc load permanent magnet generator system with multiple damper windings in the natural abc frame of reference," IEEE Trans. Energy Conversion, vol. 4, pp. 518-525, Sept. 1989.

[8] T. W. Nehl, F. A. Fouad, and N. A. Demerdash, "Determination of saturated values of rotating machinery incremental and apparent inductances by an energy perturbation method," IEEE Trans. Power App. Syst., vol. 101, pp. 4441-4451, 1982.

[9] A. A. Arkadan and B. W. Kielgas, "Effects of force fitting on the inductance profile of a switched reluctance motor," IEEE Trans. Magn., vol. 29, pp. 2006-2009, Mar. 1993.

[10] D. E. Goldberg, Genetic Algorithms in Search, Optimization and Machine Learning. Reading, MA: Addison Wesley, 1989.

[11] A. A. Arkadan, T. Sareen, and S. Subramaniam, "Genetic algorithms for nondestructive testing in crack identification," IEEE Trans. Magn., vol. 30, pp. $4320-4322$,

\section{NEED MONTH}

1994.

Lee A. Belfore, II (M'89-SM'00) received the B.S. degree in electrical engineering from Virginia Tech, BLACKSBURG, VA, in 1982, the M.S.E. degree in electrical engineering and computer science from Princeton University, Princeton, NJ, in 1983, and the Ph.D. degree in electrical engineering from the University of Virginia, Charlottesville, VA in 1990.

From 1982 to 1985, he was a Member of Technical Staff at AT\&T Information Systems NEED LOCATION. From 1987 to 1988, he was a Research Scientist in the Department of Electrical Engineering, Center for Semicustom Integrated Systems, University of Virginia. From 1990 to 1997, he was with the Department of Electrical and Computer Engineering, Marquette University, Milwaukee, WI. Since 1997, he has been with the Department of Electrical and Computer Engineering, Old Dominion University, Norfolk, VA. His research interests include neural networks, data compression, and Internet-based interactive virtual reality applications.

Dr. Before is a member of the IEEE Computer, Information Theory, Signal Processing, and System Man and Cybernetics Societies. 


\section{Abdul-Rahman}

A.

Arkadan

(S'79-M'88-SM'91-F'01) received the B.S. degree from the University of Mississippi, Starkeville, MS, in 1980, the M.S. degree from Virginia Tech, Blacksburg, VA, in 1981, and h.D. degree from Clarkson University, Potsdam, NY in 1988, all in electrical engineering.

From 1981 to 1984, he worked in industry. In 1988, he joined the Department of Electrical and Computer Engineering, Marquette University, Milwaukee, WI, as an Assistant Professor, where he is currently Professor. His interests include design, analysis, and development of electronically operated machine systems and drives, computer-aided solution of EM-field problems in EM devices, and design optimization using conventional and AI techniques.

Dr. Arkadan is a Registered Professional Engineer in the State of Wisconsin. $\mathrm{He}$ is a member of a number of the IEEE societies and active in several of its committees. He served on several Editorial Boards of IEEE conferences. He served as the Conference Chair of the 1997 First International Electric Machines and Drives Conference. He also served as the General Chair of the 2000 IEEE Conference on Electromagnetic Field Computation. 